\title{
Hybrid neural networks in cyber physical system interface control systems
}

\author{
Sergei Sokolov ${ }^{1}$, Anton Zhilenkov ${ }^{2}$, Sergei Chernyi ${ }^{3}$, Anatoliy Nyrkov ${ }^{4}$, Nikolay Glebov ${ }^{5}$ \\ $1,3,4,5$ Admiral Makarov State University of Maritime and Inland Shipping, Russia \\ ${ }^{2}$ ITMO University, Russia \\ ${ }^{3}$ Kerch State Maritime Technological University, Russia
}

\begin{tabular}{l}
\hline Article Info \\
\hline Article history: \\
Received Aug 5, 2018 \\
Revised Jan 7, 2019 \\
Accepted Dec 28, 2019 \\
\hline
\end{tabular}

\section{Keywords:}

Calculating

Complex operations

Controllers

FPGA

Programming

Simulation

\section{Corresponding Author:}

Sergei Chernyi,

Department of Electrical and Computer Engineering,

Admiral Makarov State University of Maritime and Inland Shipping,

Dvinskaya st., 5/7 Str., Saint-Petersburg, 198035, Russia.

Email: sergiiblack@gmail.com

\begin{abstract}
The calculation and results of simulation of the magnetic control system for the spacecraft momentum are presented in the paper. The simulation includes an assessment of the reliability of calculating the Earth's magnetic field parameters, as well as an assessment of the quality of object stabilization by resetting the total momentum with the aid of the system under review. The outcome of a comparative analysis of resource efficiency and energy efficiency are demonstrated in the implementation of the proposed hardware models of controllers on FPGA. The strengths and weaknesses of the programming models are shown. The developed models will allow to be modified and perform more complex operations in the future.
\end{abstract}

This is an open access article under the CC BY-SA license.

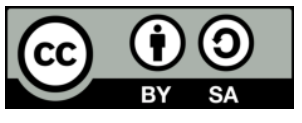

\section{INTRODUCTION}

The technology of the artificial neural network is designed to provide the machines of the future with complete autonomy, i.e. autonomous control and decision-making, relying on the previous experience and analysis without direct human involvement. Artificial neural networks, being a model of biological neural networks, are applied to solve problems, the algorithm for solving which is unknown [1-3]. As already mentioned, engine speed control can be implemented using feedback control and a PID controller. In fact, the standard PI (D) control law forms the main linear module of the vast majority of modern electronic diesel engine controllers. However, the control principle is based on the standard form of the transfer function of the propulsion system, using the transfer functions and linearization of any nonlinearities inherent in thermodynamic, physicochemical processes, for example, and combustion.

In designing the control systems for biomechanics devices, the most difficult task is the processing of pulses from the human body, recorded by means of such techniques as electromyography (EMG) and electroencephalography (EEG), and used to control, for example, prostheses, orthoses for rehabilitation purposes, exoskeletons etc. An important part of such biomechanical systems is software that processes input pulses and performs statistical processing as well as allocates patterns for creating control signals [4-5]. A neuron of the spiking neural network, as well as a system of connections of spiking neurons-spiking neural networks (NS) were selected as the object of the research for the purpose of this paper. The reasons are as follows [6-11]: 
a. Greater degree of compliance with its biological prototype from the physiological point of view

b. Information transfer by generation and propagation or absence of calibrated electric impulses with the negligible length

c. Replacement of the activation function concept by the concept of the impulse response model, (changes in the neuron's potential in time)

d. The feasibility of reconstructing a network with a dynamic structure of theoretically arbitrary volumes

e. Energy efficiency

f. Noise immunity

g. Parallel operation with multiple pulses

\section{ADVANTAGES OF THE PROPOSED APPROACH}

The first mathematical model of a biological neuron was proposed by American neurophysiologists Alan Hodgkin and Andrew Huxley, for which they were awarded with the Nobel Prize. Further, their model was simplified or refined by other researchers [10]. One of the simplest and most famous hybrid models of a biological neuron is the "integrate and work" (IF) model. This model is a model of the threshold value of the neuron, which generates a pulse at a certain voltage level. Such a model is the most computationally simple, but at the same time it is the least biorealistic. The IF model can be described by the formula [12-15]:

$$
I(t)=C_{m} \frac{d V_{m}}{d t}
$$

Where: $\mathrm{Vm}$ is the membrane potential

$\mathrm{Cm}$ is the membrane capacity

When a certain threshold value $\mathrm{Vm}$ is reached, an abrupt change in the potential occurs, which is reset and interpreted as a spike. However, if the simulated neuron does not receive a sufficient charge for the spike, then the effect of "eternal memory" appears, the charge is retained until the next pulse fires. This drawback of the IF model is eliminated by its modification, i.e. by the "integrate and operate with leakage" (LIF) model. In order to eliminate the effect of the eternal memory the value of the membrane electric resistance $\mathrm{Rm}$ is introduced [16]:

$$
I(t)=\frac{V_{m}(t)}{R_{m}}+C_{m} \frac{d V_{m}(t)}{d t} ;
$$

In order to avoid the leakage, it is required that the current value should exceed the selected threshold. The models above are neurophysiologically less approximate to reality. Such models cannot reproduce such important things as the shape of the spike, the profile of the change in the membrane potential between the spikes. However, such models are effective in computer modeling. In addition, there are further modifications to these models. Izhikevich's model is the "golden mean" between neurophysiological reliability and computational efficiency, relying on the Hodgkin-Huxley model, which reduces to a set of differential equations in a 2-dimensional space in this model [13]:

$$
\begin{aligned}
& \dot{V}=0.04 V^{2}+5 V+140-u+I \\
& \dot{u}=a(b V-u) \\
& \text { if } V \geq 30 m V \text {, then }\left\{\begin{array}{c}
V \leftarrow c \\
u \leftarrow u+d
\end{array}\right.
\end{aligned}
$$

Where: $\mathrm{V}$ is the membrane potential of the neuron $\mathrm{u}$ is the variable of the membrane potential recovery $a$ is the parameter describing the time scale of change of variable $u$ $\mathrm{b}$ is the parameter describing sensitivity of the variable $\mathrm{u}$ to subthreshold fluctuations $\mathrm{c}$ and $\mathrm{d}$ are parameters estimating the effect of currents being active in the period of the spike and afterwards

In addition, Izhikevich's model is one of the most popular today. This model is applied in the human brain project, where currently more than 1 billion neurons are modeled. 


\section{HYBRID PULSE NEURAL NETWORKS IN BIOMECHATRONIC SYSTEMS}

Often, for the development of control systems for electromechanical prosthesis, the data obtained by processing electromyograms and electroencephalograms are used. As already mentioned in the introduction, artificial neural networks are used as a classifier most often, because of the difficulties in developing computationally effective algorithms. Despite this, the artificial neural network, after training, shows high indicators of the accuracy of recognition of gestures in the analysis of EMG data. To determine two types of the hand motion (squeezing and opening) by EMG, effective algorithms have been developed that allow real-time determination of the motion type with an accuracy of 99-100\%. The algorithm provides high speed of interaction. To determine the greater number of movements, such algorithms have not been developed, so an increase in the number of detected movements leads to a decrease in quality and speed of interaction [17]. To increase the efficiency of the system, the number of electrodes is increased. This allows one to increase the accuracy of the definition of movements, improves the quality of the classifier. However, this significantly reduces the speed. The optimal number of electrodes for the prosthetic control system is one more task, which depends on many factors and requirements for the control system [17].

The primary way to improve the performance of the classifier is preliminary processing of EMG signals and extraction of its main features. This process includes segmentation, allocation of time-frequency features and diminution of dimensionality. At the same time, pre-processing has a high computational complexity. However, the choice of optimal pre-treatment methods can significantly improve the quality of the classifier performance [10-16]. The Izhikevich model is the most commonly used model among the hybrid models of spiking neural networks. It is often chosen because of its simplicity in implementation and high degree of detailing. In addition, it is quite simple to create a hardware implementation and to use it in order to control a mobile robot or an electromechanical prosthesis [15-18]. The results of using a spiking neural network show that there are no significant improvements in the quality of recognition gestures and movements [9-11]. At the same time, the computational speed is slightly lower than for classical neural networks. In addition to these popular models, there are other models. The space-time models are especially important. One can effectively visualize the results of observation and debug simulation errors with these models. For example, the space-time model of NeuCube, which simulates the behavior of 1000 neurons. This model is used to classify the patterns of hand movements using electromyography data [11]. Moreover, this model shows a high rate of learning, high accuracy of determining the patterns of hand movements, compared with other types of classifiers. However, until there is an effective algorithm for selecting model parameters that the model creators are trying to be refined [6-11].

\section{NON-INVASIVE METHODS FOR IDENTIFYING MOTOR ACTIVITY IN THE HUMAN LIMBS}

In many cases, when developing human-machine interfaces, the task is to determine the movement or intent of the movement of the human body. In particular, when developing an electromechanical hand prosthesis, at the stage of developing a prosthetic control system, the task is to identify the motor activity of the human body. To identify motor activity, various methods are used. Surface electromyography (PEMG) and electroencephalography (EEG) are traditional for this purpose. Both of these methods have their own purposes and limits of applicability. However, up to date, there are all new methods for identifying the motor activity of the human body, with the purpose and limits of applicability on their own [19-24]. Control systems based on electromyography data must possess such three important qualities of controllability as accuracy of motion detection, intuitive control and control response [25]. Of these qualities, it is only possible to quantify the accuracy of the definition of motion and the control response.

Currently, the accuracy of motion detection is quite high, exceeding 80-90\% [26-29]. However, the accuracy depends on many factors. They are as follows: the choice of the number of electrodes during the research by electromyography, the algorithms for processing the primary data of electromyography, and the choice of the classification algorithm. At the same time, it is the classifier that causes the decrease in the speed of the control system. In the classification of patterns of motion according to electromyography, neural network algorithms, neural-fuzzy logic, Support Vector Machine are used. It results in decreased control response.

Electroencephalography is a method of research the functional state of the brain, by studying its electrical activity. Electrical activity is examined by an electroencephalograph device that receives and converts signals taken by electrodes on the surface of the human head according to the international system "10-20\%" [30-32]. The electroencephalogram obtained by this method is integral throughout the neurophysiological activity of the human body, being an indicator of the quality of the ongoing neurophysiological processes. Signals from the skull are recorded by means of encephalography. Further, the signal is digitized, processed and used in device control systems [15-19]. 
In addition to the use of the encephalography method in BCI systems, its application is developing in the research of neural feedback. In this case, the data on the electrical activity of the brain is used when trying to improve the effectiveness of human activity (increase attention, improve concentration, etc.), in treatment and diagnosis [15-20]. There are prospects for using neural feedback in training to control brain-computer interfaces.

The task of processing electroencephalograms and identifying cognitive patterns is much more difficult than processing the data of electromyography. The accuracy of the definition of motion, even simple (opening-closing eyes, squeezing-opening the hand) is much lower than in the classification of patterns of electromyography $[13,28-32]$. However, the prospects of using electroencephalography are higher, since there are opportunities to determine the patterns of intentions of movement.

\section{VIRTUAL MODELING}

The method of optical myography is one among such non-invasive methods. The method is based on the visual definition of muscle contraction and relaxation with the use of a camera and technical vision techniques. The model of neurons performs the calculation of the recovery, the membrane potential, the processing of the pulse queue and the generation of new impulses. Each neuron has a vector of presynapses and postsynapses, as well as a vector of pulses with the parameters of the current and the time interval required to trigger the pulse.

Moreover, the weight of the synapse is changed after a certain period of time. Setting the interval of updating the weight of the synapse allows one to monitor and change the frequency of weight updates. The mathematical LIF model has only one differential equation [1-10]:

$$
\begin{aligned}
& \frac{d v}{d t}=I+a-b v, \\
& \text { if } v \geq v_{\text {thresh }} \text { then } v \leftarrow c .
\end{aligned}
$$

The neuron mathematical model by Izhikevich can be written as follows:

$$
\begin{aligned}
& \dot{v}=0.04 v^{2}+5 v+140-u+I, \\
& \dot{u}=a(b v-u), \\
& \text { if } V \geq m V, u \leftarrow c, u \leftarrow u+d, \\
& \text { where }=\frac{d}{d t^{2}} .
\end{aligned}
$$
see in Figure 1.

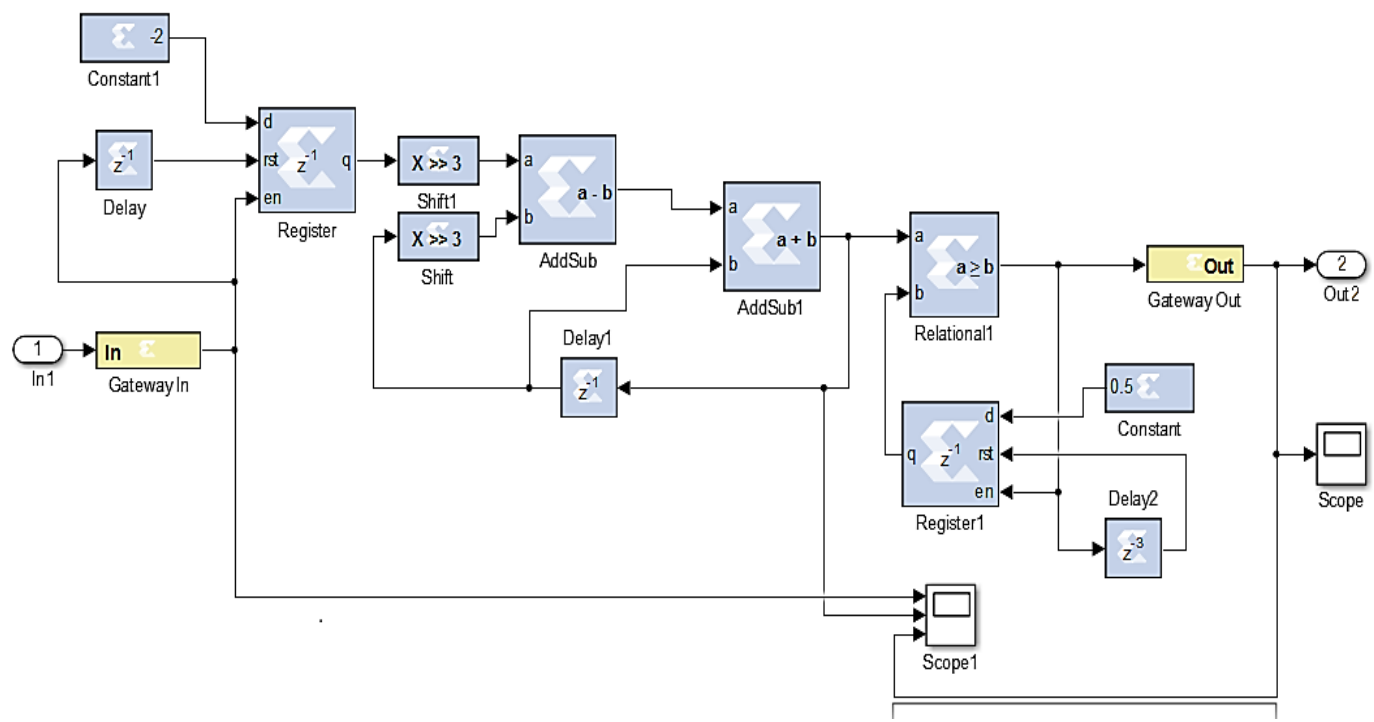

Figure 1. The model of the spiking artificial neuron leaky integrate and fire (LIF) 
Izhikevich's neuron model is more complicated can see in Figure 2. The Subsystem block is a neuron soma, the queue of impulses is processed there. Further, the integrators are the pulse filter blocks, they determine if the pulse is exciting or not [20-25]. The computing center of Izhikevich's neuron is shown in Figure 3. In Izhikevich's model, on the contrary, the voltage drop occurs not at the threshold, but at the peak of the pulse that shown in Figure 4.

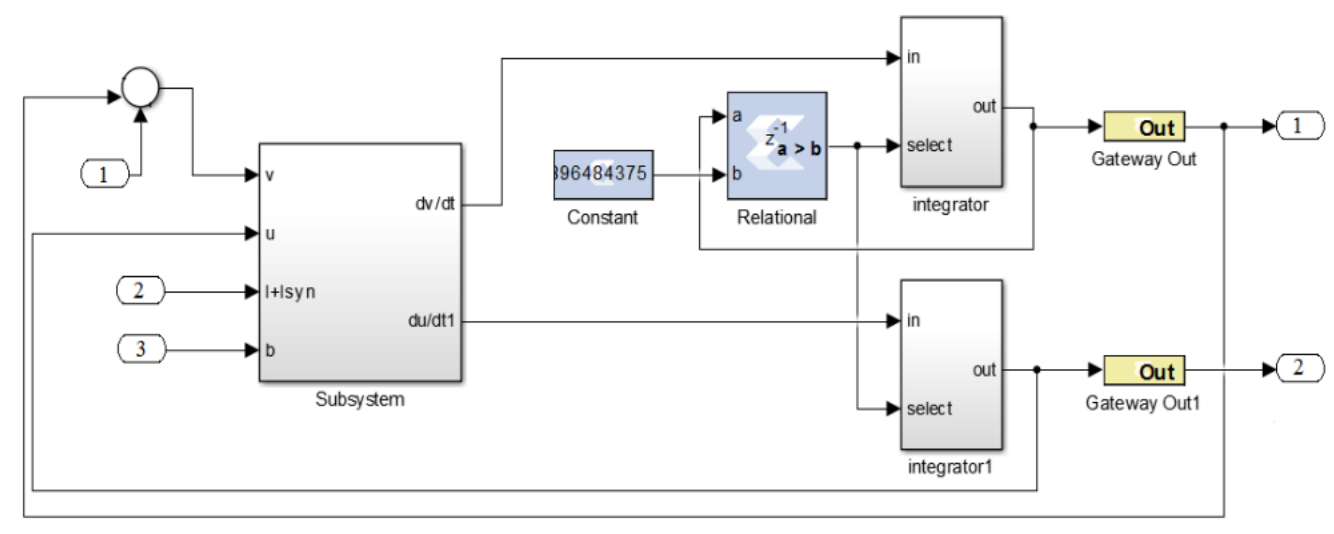

Figure 2. Izhikevich's spiking artificial neuron model

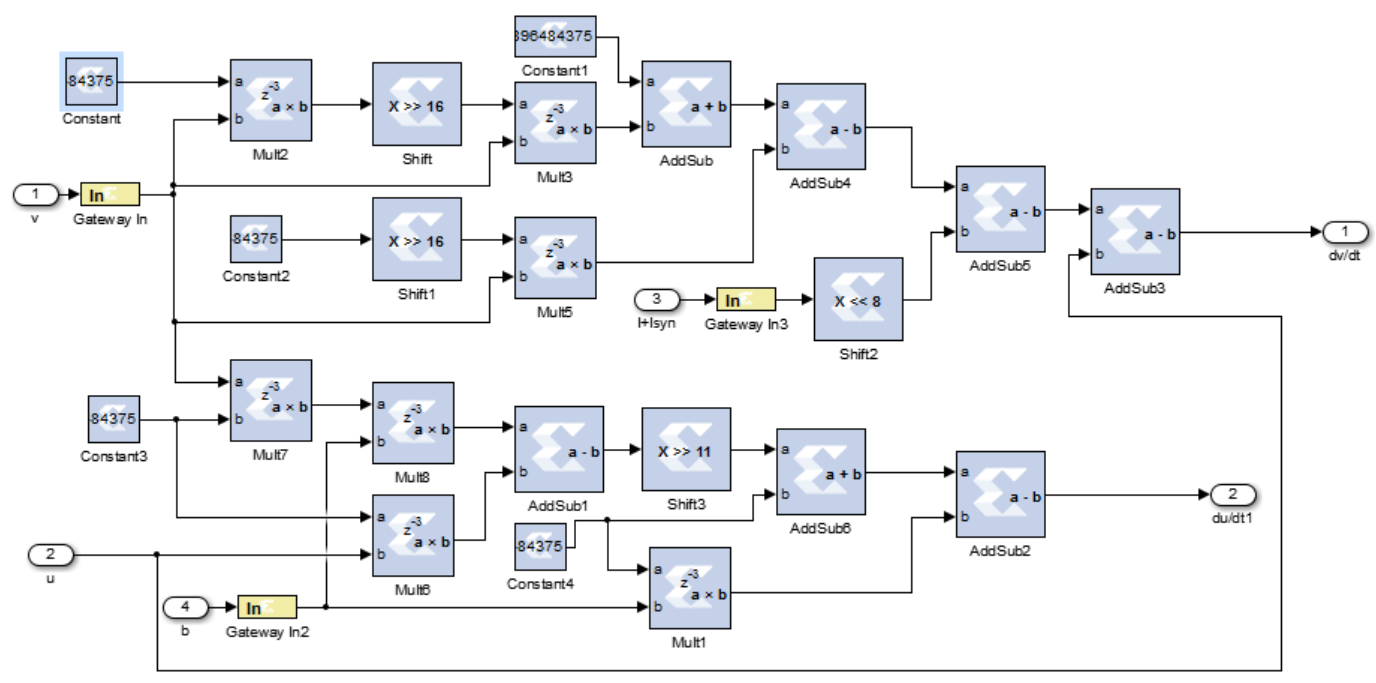

Figure 3. Izhikevich's spiking artificial neuron model

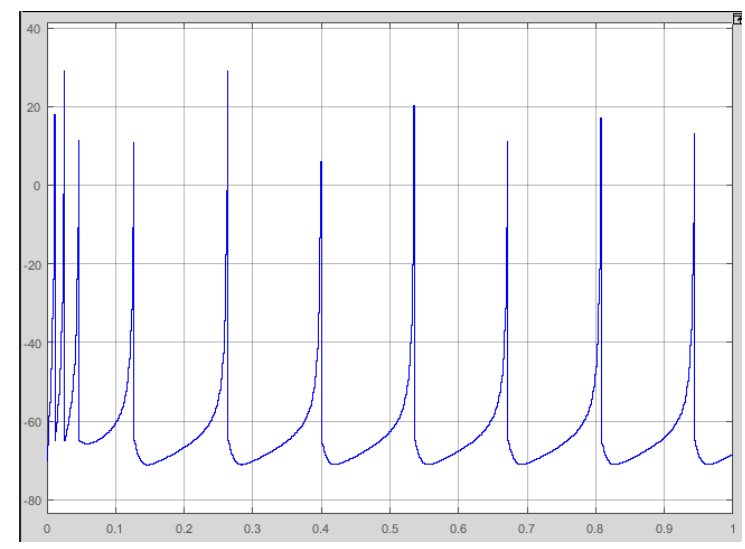

Figure 4. Izhikevich neuron model membrane voltage 
This function is formed by adding synapse and training modules (STDP) can see in Figure 5. An example of implementing a mobile robot control system based on developed models is shown in Figure 6. Model is generated from the first model of the LIF-neuron (Figures 5 and 6), due to the inclusion of the STDP module in the model.

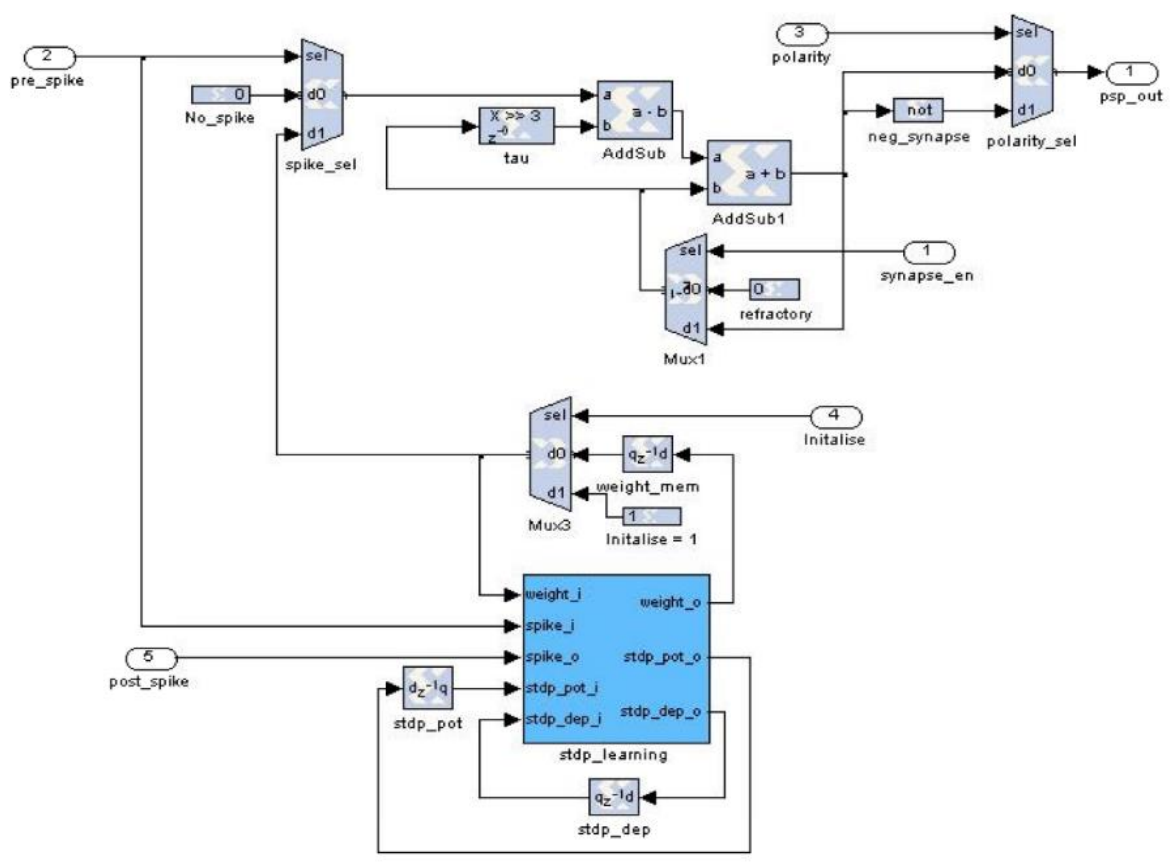

Figure 5. Self-learning model of spiking artificial neuron leaky integrate and fire

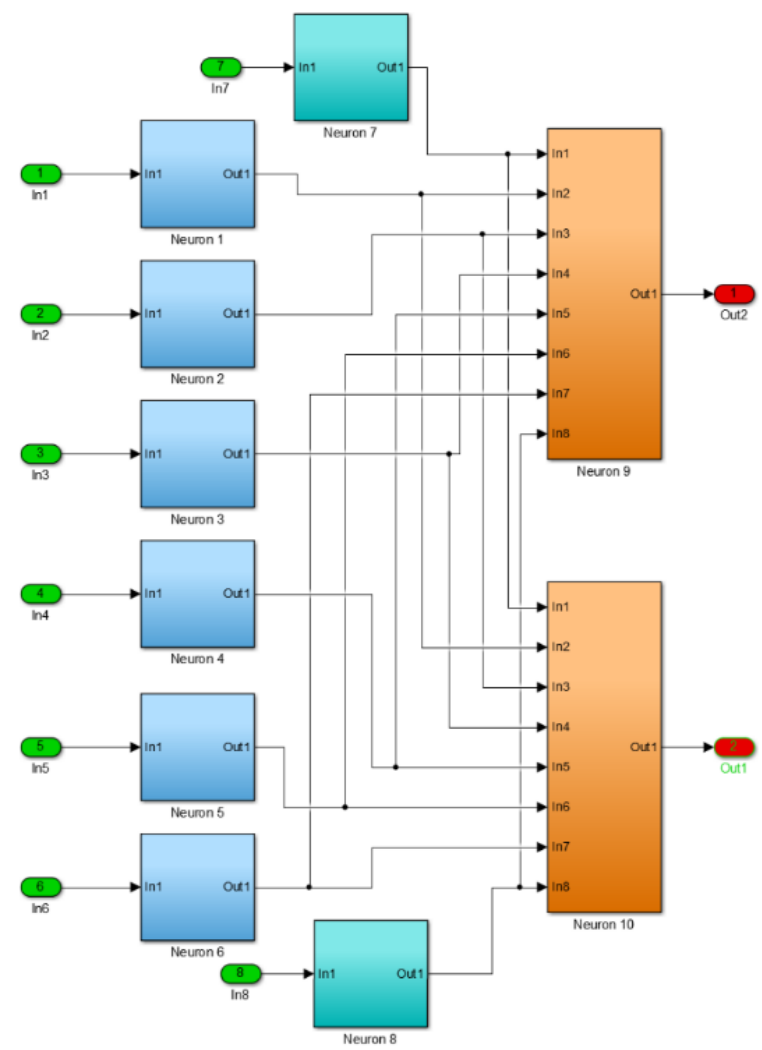

Figure 6. An example of implementing a mobile robot control system based on developed models 


\section{CONCLUSIONS}

The spiking neural networks by Izhikevich's model is more bio-realistic. When developing human-machine interfaces, a technique with ultrasonic sensors is also used. This method is increasingly gaining popularity, because due to it, it becomes possible to examine the morphological structure of the human limb in more details, having received an image for processing. This makes it possible to find out what muscle is contracts and relaxes at the time moment. In comparison with the method of surface electromyography, this method with ultrasonic sensors shows a higher accuracy when using the same classification algorithms. However, now this method has significant drawbacks, which do not allow creating the human-machine interfaces based on it.

\section{REFERENCES}

[1] Rutkowska D., et al., "Neural networks, genetic algorithms and fuzzy systems," Telecom, 2006.

[2] W. Gerstner and W. M. Kistler, "Spiking neuron models: Single neurons, populations, plasticity," Cambridge, Cambridge University Press, 2002.

[3] E. M. Izhikevich, "Dynamical systems in neuroscience: The geometry of excitability and bursting," The MIT Press, 2007.

[4] K. Ahmed, et al., "Probabilistic inference using stochastic spiking neural networks on a neurosynaptic processor," 2016 International Joint Conference on Neural Networks (IJCNN), pp. 4286-423, 2016.

[5] A. Nyrkov, et al., "Mathematical models for solving problems of reliability maritime system," Advances in Systems, Control and Automation, pp. 387-394, 2018.

[6] A. Nyrkov, et al., "Identification and tracking problems in qualimetry inspections in distributed control systems of drilling platforms," 2016 IEEE NW Russia Young Researchers in Electrical and Electronic Engineering Conference (EIConRusNW), St. Petersburg, pp. 641-645, 2016. DOI: 10.1109/EIConRusNW.2016.7448265

[7] D. M. Malioutov, et al., "Covariance matrix estimation for interest-rate risk modeling via smooth and monotone regularization," IEEE Journal of Selected Topics in Signal Processing, vol. 10, pp. 1006-1014, 2016.

[8] A. Chaudhuri and H. Stenger, "Survey sampling theory and methods," 2nd Edition, CRC Press, 2005.

[9] N. Elia, "When Bode meets Shannon: control-oriented feedback communication schemes," IEEE Transactions on Automatic Control, vol. 49, no. 9, pp. 1477-1488, 2004.

[10] C. E. Shannon, "A mathematical theory of communication," The Bell System Techical Journal, vol. 27, no. 3, pp. 379-423, 1948.

[11] J. P. Hespanha, et al., "A survey of recent results in networked control systems," Proceedings of the IEEE, vol. 95, no. 1, pp. 138-162, 2007.

[12] N. Elia, "Remote stabilization over fading channels," Systems and Control Letters, vol. 54, no. 3, pp. 237-249, 2005.

[13] A. Nyrkov, et al., "the use of fuzzy Neural Structures to Increase the Reliability of Drilling Platforms," Proceedings of the 26th International DAAAM Symposium, pp. 0672-0677, 2016.

[14] A. Zhilenkov and S. Chernyi, "Investigation performance of marine equipment with specialized information technology," Procedia Engineering, vol. 100, pp. 1247-1252, 2015. DOI: 10.1016/j.proeng.2015.01.490

[15] W. Maass and C. M. Bishop, "Pulsed neural networks," The MIT Press, 1999.

[16] W. Maass, et al., "On the computational power of sigmoid versus boolean threshold circuits," Proceedings of the 32nd Annual Symposium on Foundations of Computer Science, pp. 767-776, 1991.

[17] D. Abdelghani and A. Boumediène, "Direct torque control of two induction motors using the nine-switch inverter," International Journal of Power Electronics and Drive Systems (IJPEDS), vol. 9, no. 4, pp. 1552-1564, 2018.

[18] M. Huang and S. Dey, "Dynamic quantization for multisensor estimation over bandlimited fading channels," IEEE Transactions on Signal Processing, vol. 55, no. 9, pp. 4696-4702, 2007.

[19] E. J. Msechu, et al., "Decentralized quantized Kalman filtering with scalable communication cost," IEEE Transactions on Signal Processing, vol. 56, no. 8, pp. 3727-3741, 2008.

[20] I. F. Akyildiz, et al., "A survey on sensor networks," IEEE Communication Magazine, vol. 40, no. 8, pp. 102-114, 2002.

[21] K. You, et al., "Asymptotically optimal parameter estimation with scheduled measurements," IEEE Transactions on Signal Processing, vol. 61, no. 14, pp. 3521-3531, 2013.

[22] J. Wu, et al., "Event-based sensor data scheduling: trade-off between communication rate and estimation quality," IEEE Transactions on Automatic Control, vol. 58, no. 4, pp. 1041-1046, 2013.

[23] G. Battistelli, et al., "Data-driven communication for state estimation with sensor networks," Automatica, vol. 48, no. 5, pp. 926-935, 2012.

[24] C. Wikle and N. Cressie, "A dimension-reduced approach to space-time Kalman filtering," Biometrika, vol. 86, no. 4, pp. 815-829, 1999.

[25] K. Plarre and F. Bullo, "On Kalman filtering for detectable systems with intermittent observations," IEEE Transactions on Automatic Control, vol. 54, no. 2, pp. 386-390, 2009.

[26] Y. Mo and B. Sinopoli, "Towards finding the critical value for Kalman filtering with intermittent observations," arxiv: 1005.2442, 2010.

[27] M. Huang and S. Dey, "Stability of Kalman filtering with Markovian packet losses," Automatica, vol. 43, no. 4, pp. 598-607, 2007.

[28] L. Xie and L. Xie, "Stability of a random Riccati equation with Markovian binary switching," IEEE Transactions on Automatic Control, vol. 53, no. 7, pp. 1759-1764, 2008. 
[29] K. You, et al., "Mean square stability for Kalman filtering with Markovian packet losses," Automatica, vol. 47, no. 12, pp. 2647-2657, 2011.

[30] M. Trivellato and N. Benvenuto, "State control in networked control systems under packet drops and limited transmission bandwidth," IEEE Transactions on Communications, vol. 58, no. 2, pp. 611-622, 2010.

[31] M. Es-Saadi, et al., "using the five-level npc inverter to improve the FOC control of the asynchronous machine," International Journal of Power Electronics and Drive Systems (IJPEDS), vol. 9, no. 4, pp. 1457-1466, 2018.

[32] A. Hamed and A. Hazzab, "Modeling and real-time simulation of induction motor using RT-LAB," International Journal of Power Electronics and Drive Systems (IJPEDS), vol. 9, no. 4, pp. 1476-1485, 2018.

\section{BIOGRAPHIES OF AUTHORS}
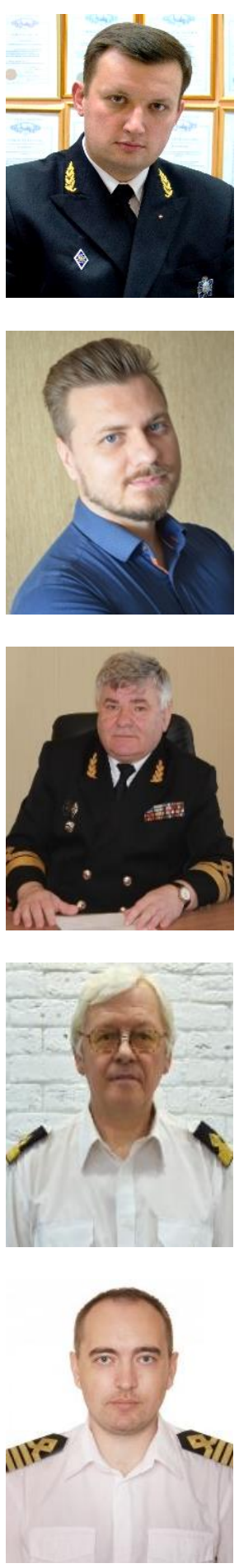

Sergei Sokolov. PhD, Professor

Department of Integrated Information Security

Admiral Makarov State University of Maritime and Inland Shipping

198035, Russia, Saint-Petersburg, Dvinskaya st., 5/7

Contact us: +7 (812) 748-96-92

sokolovss@gumrf.ru

Research interests: neural systems, marine vessels, decision making, mathematical optimization. The author of more than 100 scientific papers in highly rated journals.

Anton Zhilenkov, PhD

ITMO University, Kronverkskiy prospect, 49, Saint Petersburg, 197101, Russia

zhilenkovanton@gmail.com

Research interests: neural systems, mathematical optimization.

The author of more than 150 scientific papers in highly rated journals.

\section{Nikolay Glebov, $\mathrm{PhD}$}

Department of Integrated Information Security

Admiral Makarov State University of Maritime and Inland Shipping

198035, Russia, Saint-Petersburg, Dvinskaya st., 5/7

Contact us: +7 (812) 748-96-92

otd_o@gumrf.ru

Research interests: marine vessels. The author of more than 100 scientific papers.

Anatoliy Nyrkov, PhD, Professor

Department of Integrated Information Security

Admiral Makarov State University of Maritime and Inland Shipping

198035, Russia, Saint-Petersburg, Dvinskaya st., 5/7

NyrkowAP@gumrf.ru

Research interests: neural systems, marine vessels, decision making, mathematical optimization.

The author of more than 200 scientific papers in highly rated journals.

\section{Sergei Chernyi, $\mathrm{PhD}$}

Department of Integrated Information Security

Admiral Makarov State University of Maritime and Inland Shipping

198035, Russia, Saint-Petersburg, Dvinskaya st., 5/7

Contact: sergiiblack@gmail.com

Research interests: mathematical optimization.

The author of more than 100 scientific papers in highly rated journals. 